\section{Simulating impairment through virtual reality}

\author{
Jacqueline Mclntosh, Bruno Marques \\ and Robyn Harkness
}

International Journal of

Architectural Computing $1-12$

(C) The Author(s) 2020

Article reuse guidelines: sagepub.com/journals-permissions DOI: 10.1 I77//478077/209/4020 journals.sagepub.com/home/jac @SAGE

\begin{abstract}
Research on architectural technology for health care has rapidly increased in recent years; however, little research has been conducted on the use of virtual reality for simulating impairment. This exploratory research maps the experiences of people with impairments in the often-overlooked corridors and waiting rooms of an emergency department. It questions whether the experience of an impairment can be usefully simulated for empathetic design. While using participatory processes to develop a virtual reality simulation of waiting areas, this research applies three representative impairments and then surveys 30 architectural designers to find the emotional responses of the unimpaired to the design intervention. While this research is preliminary, it is particularly valuable for the comprehension of proposed designs during the early planning and design phases, without costly and time-consuming use of full participatory processes. It finds there is significant potential for the use of virtual reality as a technology to simulate the experiences of these spaces by individuals with impairment, enabling empathetic design, and offers direction for future research.
\end{abstract}

\title{
Keywords
}

Emergency department, virtual reality, architecture, participatory design, health care

\section{Introduction}

Emergency departments (EDs) worldwide provide care and treatment for patients with real or perceived serious injuries or illnesses, with over half of these incidents being deemed potentially life-threatening. In New Zealand, there is an increasing demand for more, larger, more productive EDs. Overcrowding and delays to treatment first emerged as a problem in New Zealand in the mid-1990s. ${ }^{1}$ Today, District Health Boards around the country are struggling more than ever to meet demands reporting record numbers of patients. ${ }^{2}$ In the future, this situation is predicted to worsen with the increasing demand associated with growing and ageing populations as well as incidences of long-term conditions and challenges in aged residential care management. ${ }^{3}$ Among the highest of demographics attending EDs are those experiencing disability. ${ }^{4}$

School of Architecture, Victoria University of Wellington, Wellington, New Zealand

\section{Corresponding author:}

Bruno Marques, School of Architecture, Victoria University of Wellington, Wellington 6I40, New Zealand.

Email: bruno.marques@vuw.ac.nz 
In 2018, an estimated $22.3 \%$ of people living in New Zealand were identified as disabled, an increase of $20 \%$ from $2001 .{ }^{5}$ Certain impairments are more likely than others to impact an individual's ability to engage with the physical environment, including those that affect the perceptual systems and influence how the users experience space. People with these conditions are typically in higher need of emergency health care but limited in their abilities to attend such places.

The design of EDs constitutes one of the greatest threats to quality of emergency care. ${ }^{6-8}$ Poor design is associated with increased risk of error, delayed time-critical care, increased morbidity and excessive number of deaths. ${ }^{9,10}$ This is exacerbated in EDs where high levels of artificial lighting, institutional furnishings, chaotic activity, alarming noises and cavernous rooms are considered standard. ${ }^{11}$ These lead to increases in anxiety, delirium and high blood pressure. ${ }^{12}$ Progressively, greater attention has been paid to the psychological and emotional risks involved in health care environments and the needs of the first-hand user; however, while this relationship has been extensively investigated with respect to demographics, such as age and gender, there is very limited evidence in the health care literature that acknowledges the needs and emotions of users with sensory impairments or disability, ${ }^{13}$ whether temporary or permanent.

Almost all of these negative impacts occur in the interstitial spaces of EDs - the corridors and hallways where users spend long periods of time 'between-moments', waiting for treatment. ${ }^{14}$ Despite the conditions of use for these circulation spaces, little consideration is typically given to their design, and most fall into extremes of either under- or over-stimulation for the individual users. EDs are places where users are experiencing a very broad spectrum of emotions, ranging from boredom, to anxiety, to fear at any given moment. The architecture and occupancy conditions are either unable to sufficiently stimulate those users with reduced senses for prolonged periods of time or they become overwhelming for those with intensified senses. ${ }^{15}$

The architectural design tools and devices to explore highly charged sensory spaces, such as those in emergency rooms, have been historically limited. Virtual reality (VR) is emerging as a powerful threedimensional technology, offering designers the opportunity to comprehend proposed designs more clearly during the planning and design phases. The use of VR can allow greater influence on design decision-making as well as facilitate communal and recreational designs that need greater participatory input from architects, clients and users in a collaborative manner. ${ }^{16}$ Many studies conducted ${ }^{16-18}$ report that virtual environments allowed the evaluation of unnecessary design environments and the development of collaborative multi-participant settings to improve the quality of conceptual and schematic designs, and improved the design quality of the implemented design.

Although computer-generated, VR closely resembles reality and allows the sharing of a single 'reality' by multiple people, ${ }^{19,20}$ making the design process engaging, interactive and with a high degree of realism; ${ }^{18}$ more importantly, it allows the researcher to isolate and modify variables in a controlled environment, facilitating participation or even co-design with stakeholders in a cost-effective manner. ${ }^{20}$ Evidence that the experience is 'real' can be found in the numerous studies reporting emotional engagement. ${ }^{21-23}$ However, very few studies in VR thus far have focused on the qualitative aspects of architectural spaces, exploring the psychological and physical dimensions of light, materiality, colour, form and texture. Moreover, VR experiments for those impaired have produced experiences that either rely on pure auditory interfaces with limited competence in describing the shape and form of a specific virtual layout or provide only haptic feedback based on stationary controllers, ${ }^{24,25}$ both limiting their mobility in a virtual environment and the perception of the virtual space. This presents a limitation towards the importance of subjective meanings and experiential aspects in space-making. ${ }^{26,27}$

This research explores the potential for VR using participatory processes in communicating design input from the primary occupants (impaired patients) to the designers, during all phases of design. It examines how VR could be used to understand the critical phenomenology and qualities of these interstitial health care places in relation to the impairments, typically experienced by the most predominant ED users, and uses these findings to address the challenges of ED architecture. 
Table I. Personas.

\begin{tabular}{lllll}
\hline Name & Impairment & Sex & Age & Cause of impairment \\
\hline Olivia & Autism spectrum disorder & Female & 10 & Existed at birth \\
Bernie & Deaf & Male & 75 & Age related \\
Eliza & Visual & Female & 21 & Eye disease \\
\hline
\end{tabular}

\section{Method}

The research employs a VR simulation of ED interstitial spaces,${ }^{28}$ using personas and narrative, and manipulating spatial variables to influence emotions. Assessment of spatial qualities of architectural design in virtual settings can involve both qualitative and quantitative factors. Qualitative aspects may expand to affective and environmental qualities of space, while quantitative measurements may include scale and distance, proportions and size. ${ }^{26}$ The well-known model to quantify emotional responses and environmental preferences $^{29}$ uses a non-numeric scale based on adjectives to describe aspects of a certain environment, ${ }^{29,30}$ commonly used in environmental psychology. These adjectives are an effective measure to understand both emotional and physical meanings of a certain architectural space, conveying aspects of size, temperature, colour, materiality, style and form. This model is also effective as a way to compare qualitative virtual perceptions with real-world perceptions to find similarities and discrepancies in both environments. ${ }^{31}$

In order to utilise VR as a tool for design, an advanced method was required. First, a set of personas were developed with the assistance of disability associations to represent the impairments most commonly experienced in an ED setting. Second, a narrative was mapped with the assistance of hospital personnel, from the patient's arrival at the ED until their departure. Periods of waiting were identified along with their locations and characteristics. A simplified hospital ED was then modelled three-dimensionally. Participants with impairment were surveyed to obtain their experience of these spaces. Then, the experiences of impairment were used to modify aspects of the emergency waiting space designs, such as size, temperature, colour, materiality, style and form. Finally, the modified interiors were tested by designers empathetically to determine whether the emotions solicited were representative of the impaired participants.

Participation was used during three stages of the process: working with health care professionals to develop the narrative of the ED, interviewing people with impairments to develop the VR simulations and finally testing the simulations on design participants to determine their effectiveness.

\section{Personas}

Three personas, as shown in Table 1, were developed based on recorded interviews with individuals with the named impairment most prevalent in the location of the subject hospital. The number of people with impairments interviewed ranged between 4 and 7 per impairment. Traits such as gender, age and reason for impairment were developed as representative of the wider demographic. The impairments selected were hearing impaired, sight impaired and autism spectrum disorder. Impaired participants were recruited through organisations established for their impairment, for example, the Blind Foundation, to aid in the development of the personas. All participants were above the age of 18 and eligible to sign the consent form or provide verbal recorded consent (Human Ethics Approval No. 0000026043, Victoria University of Wellington). Through interviews, environmental settings were established and each of the personalities was given a full backstory in order to allow design participants to comprehend their world. Environmental settings included adjusting focus, altering visual information and visually stimulating audio effects in order to describe the unique sensory conditions of each impairment. ${ }^{32}$ Interviewees with impairments were exposed to both the simulation and the representation of their impairment and allowed to make changes. Where the impairment made this 


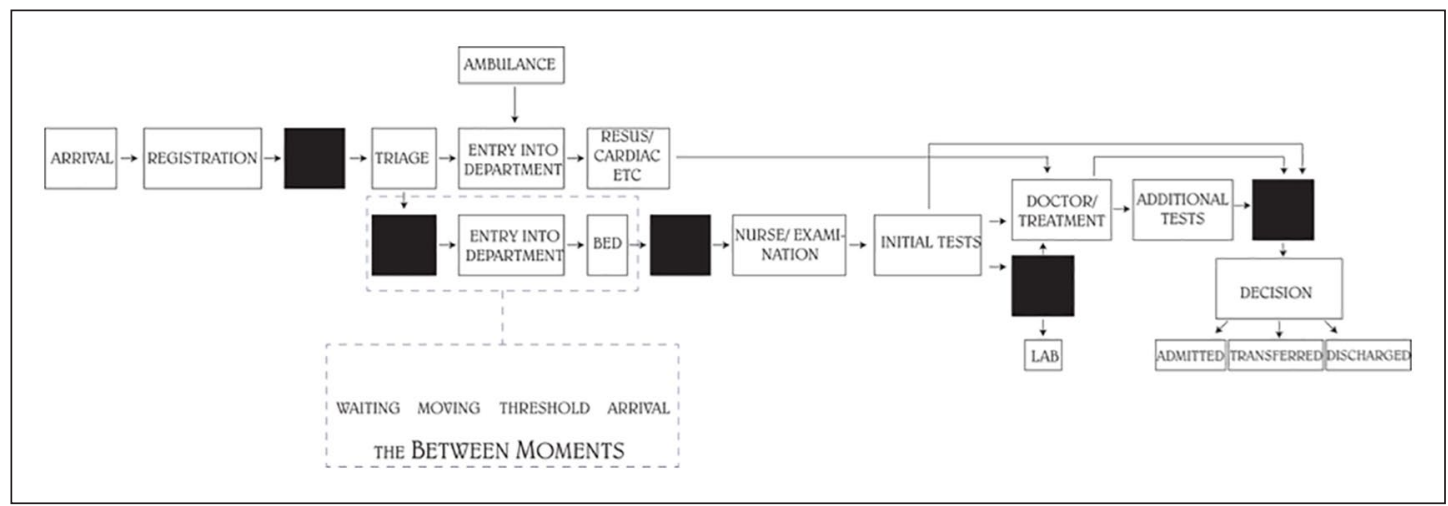

Figure I. Emergency department narrative.

Table 2. Variables.

\begin{tabular}{llllll}
\hline Area & & Variables & & \\
\hline Wall & Concrete & Brick & Wood & Glass & Plastic \\
Floor & Concrete & Wood & Carpet & & \\
Ceiling & Tile & Wood & Metal & \\
Light & Colour $(\mathrm{K})$ & & & \\
\hline
\end{tabular}

difficult, such as for those visually impaired but not fully blind, an extended dialogue was used between the impaired individual for making changes. Finally, the designs were modified by the researchers based on the feedback from the impaired participants, which then allowed design participants to experience variables from three different points of view.

\section{Narrative}

In order to develop the hypothetical ED simulation, it was imperative to understand the narrative - the process that a patient follows upon entering the space, moving, crossing thresholds and arriving, in order to analyse the sensory conditions in areas of high stress or high monotony. The narrative was mapped with the assistance of hospital personnel, from the patient's arrival at the ED until their departure. Incidences of between-moments are identified by black squares in Figure 1. To reduce the scope of the study, a key phase was identified where the patient moves from the public waiting room to their bed (in dashed box in Figure 1). In this short phase, the patient experiences waiting, movement, a threshold and a moment of arrival, allowing for a broad range of emotional responses. This phase is the section of the ED that has been simulated.

\section{Variables}

Dependent variables (Table 2) which can alter the experience of a space were identified in common building materials and characteristics of light, which affect human perceptual systems and the emotional responses for each of the personas. ${ }^{31,33}$ These variables initially included the texture and colour of wall, floor and ceiling, and the lighting and shape and size of the room. ${ }^{34}$ Following the completion of a pilot study, these 


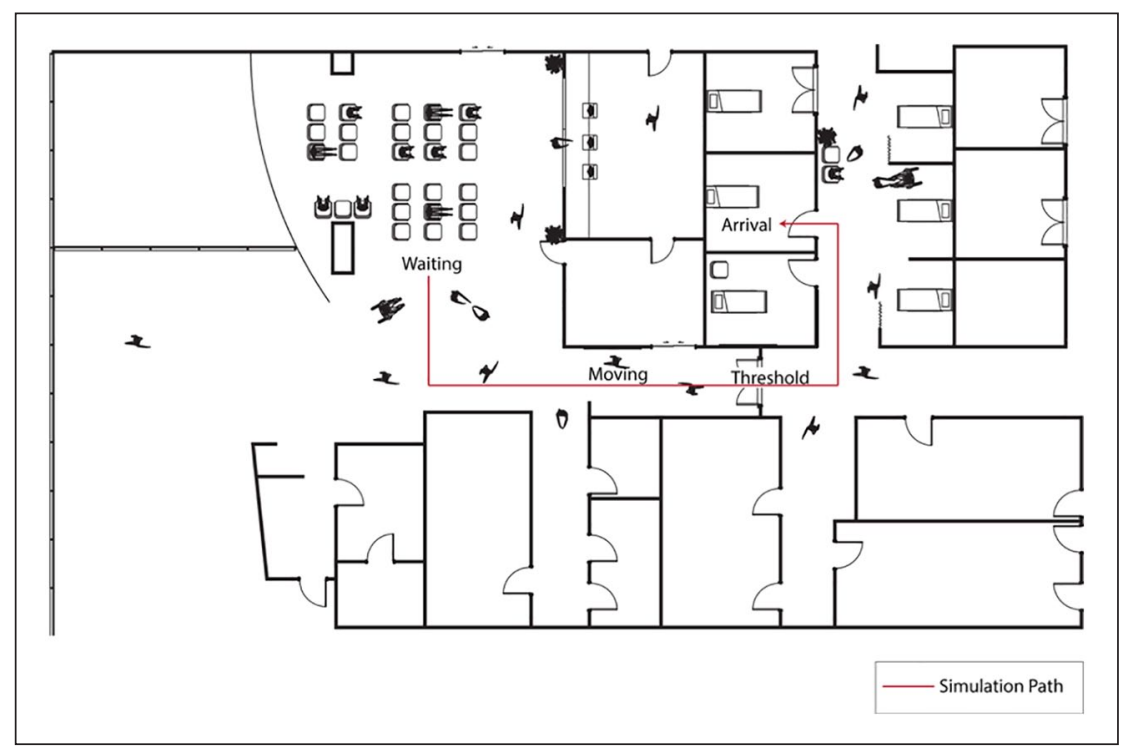

Figure 2. Plan of Simulation I (existing ED).

variables were reduced to include the colour and texture of the walls, ceilings and floors and the lighting colour. Three colour options were offered for each material.

\section{Participants}

The process was tested in a pilot study using six participants. By using a small number of participants, testing and validating of the method were undertaken prior to the larger experiment and presented for discussion at a conference. ${ }^{35}$ For the final VR experiment, 30 non-impaired architectural designers were selected to participate in the study to understand how the VR tool affected their understanding of the design. Each persona was experienced by a group of 10 participants. They were recruited voluntarily through architectural networks and professional bodies via online registration.

\section{Materials}

Equipment and software. The VR equipment included an HTC Vive with a $110^{\circ}$ field of view and a tracking area of $5 \times 5 \mathrm{~m}^{2}$. An Alienware Aurora R5 processor was connected to the VR equipment. Steam VR and Fuzor 2018 were used to run the experiment.

Simulation space was modelled with four elements: waiting, movement, a threshold and a destination (Figure 2). A single lighting system with constant brightness was chosen to create consistent light dispersal over all spaces, although the level of lighting changes between personas.

\section{Procedure}

Design participants were individually introduced to the VR laboratory and provided with a list of 24 positive and 24 negative emotions to choose from (Table 3). Each participant was then told the background story of their persona, including information about the disability, why they were at the ED, how long they had been 
Table 3. Emotions.

\begin{tabular}{llllll}
\hline Negative & & & Positive & & \\
\hline Disappointed & Envy & Fear & Kindness & Lust & Sympathy \\
Insecurity & Contempt & Indignation & Admiration & Relief & Amused \\
Shame & Regret & Pity & Energised & Courage & Respect \\
Anxiety & Confusion & Shock & Enchanted & Desire & Confident \\
Doubt & Distress & Sadness & Fascination & Hope & Euphoria \\
Loneliness & Anger & Frustration & Inspired & Pride & Surprise \\
Annoyance & Embarrassment & Disgust & Anticipation & Satisfied & Worship \\
Dissatisfied & Boredom & Guilt & Enthusiasm & Joy & Confidence \\
\hline
\end{tabular}

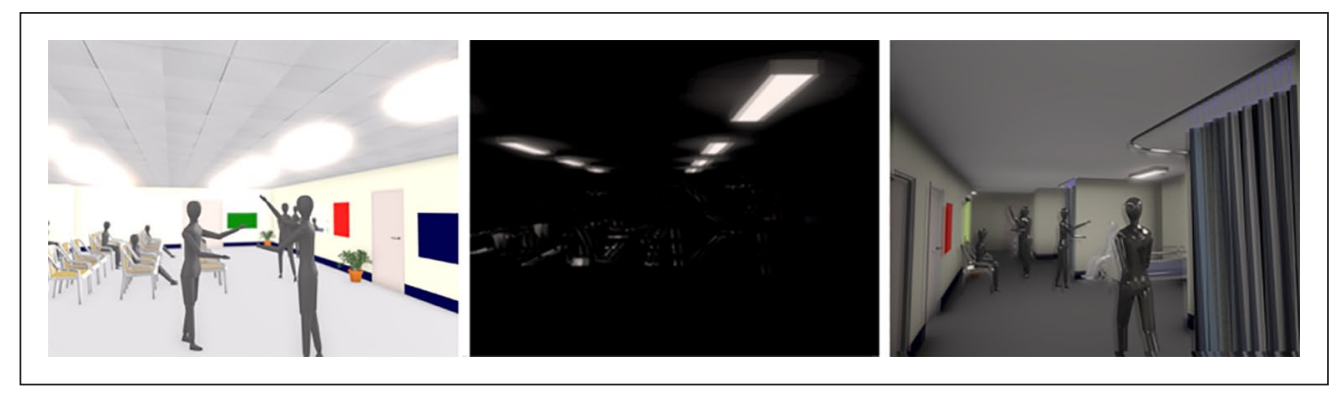

Figure 3. Simulation I (existing ED), from left: Olivia, Eliza and Bernie.

there for and who they were with. The experiment process was explained and the subjects entered into the simulation of an existing ED (Figures 2 and 3), beginning in the waiting space. A two-staged simulation process was adopted to confirm that the experiences of the VR simulation were similar to those of the impaired participants and to identify those design variables with the most impact on the impairment. In the first simulation, participants were asked to describe any negative emotions that their persona might experience in the space and what positive emotions they would instead like the architecture to influence. This was repeated for each of the moments, including moving (walking down a hallway), thresholds (going through a doorway) and arrival (reaching the room they will be treated in).

The second simulation (Figures 4 and 5) brought design participants into a hypothetical ED space. In this simulation, still experiencing the space as one of the personas, participants were asked to select the design variables for the walls, floor, ceiling and light that most influenced their selected positive emotion from the previous simulation. This was repeated as they progressed from waiting to moving through a threshold and then reaching the moment of arrival.

\section{Findings}

Results from the study showed a clear relationship between the absence of a perceptual system (impairment), the individual's perception of space and the influence the architectural design variables had on user emotions. Figure 6 shows the adjectives that describe the emotions experienced by the personas at each stage of the narrative, as identified by the group of 10 designers. The study found that participants initially felt emotions such as anxiety, fear and confusion within the 'between-moments' of the ED narrative, but hoped for architecture that would instead influence calmness, relaxation, relief and hope during the moments of 


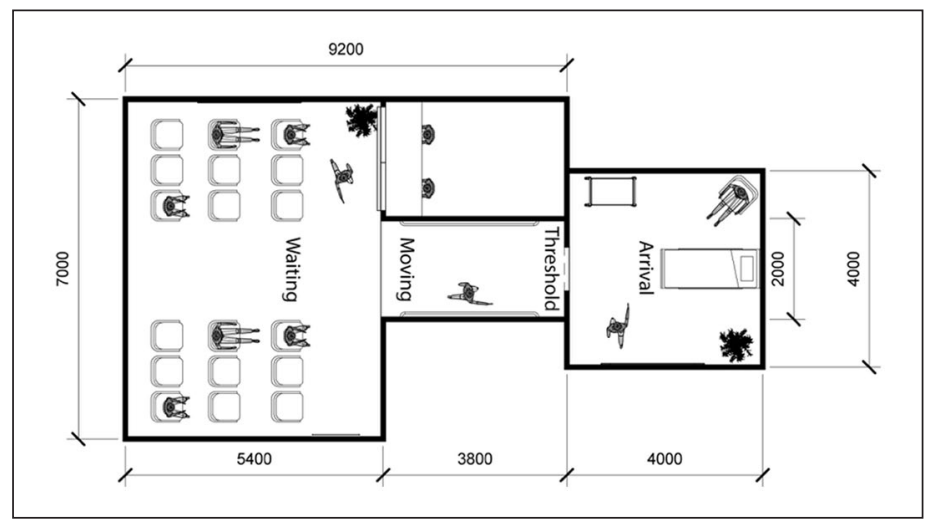

Figure 4. Plan of Simulation 2 (hypothetical ED).

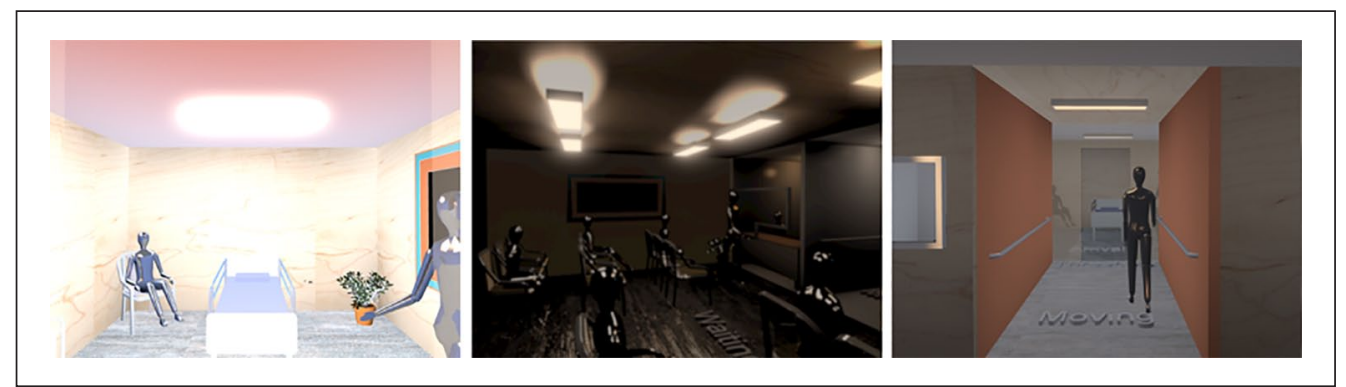

Figure 5. Simulation 2 (hypothetical ED), from left: Olivia, Eliza and Bernie.

waiting and arrival. These results were compared with the emotion survey of the impaired participants. The results showed that despite impairment, largely all participants hoped to feel confident while in the moments of movement and thresholds. The emotional response described by the participants increased their sense of presence in the virtual environment.

In order to influence these emotions, relationships were found between the emotion and the variable choices, with the opinions changing between personas. More specifically, when participants were experiencing Eliza, the visually impaired simulation, they found that lighting on more reflective surfaces, such as glass and metal, gave her visual information, increasing the sense of joy and decreasing the sense of anxiety and fear. The carpet was the predominant material chosen for Eliza as it reduced the noise of surrounding footsteps and echoes, decreasing participant confusion. Similarly for Bernie, quiet spaces with simple signage and a direct viewshaft to the reception from the waiting area and main entrance were preferred as they limited confusion and chaos. The use of red on negative corners and green on positive corners facilitated easy navigation and provided confidence and hope for those experiencing Bernie. When experiencing the spaces as Olivia, light was seen with greater intensity. Participants found this increasingly apparent with light of 5000-6000 K (fluorescent lighting), lighting which is commonly found in hospital environments. This lighting quality increased participants' feelings of anxiety due to the perception of overwhelming amounts of information. By changing the lighting to a medium yellow tone, the experienced emotion changed to one of courage.

Concerning the colour of the wall, floor and ceiling, the variable mainly selected was one of personal preference of the participant rather than the selection of colour that influenced a specific emotion. 


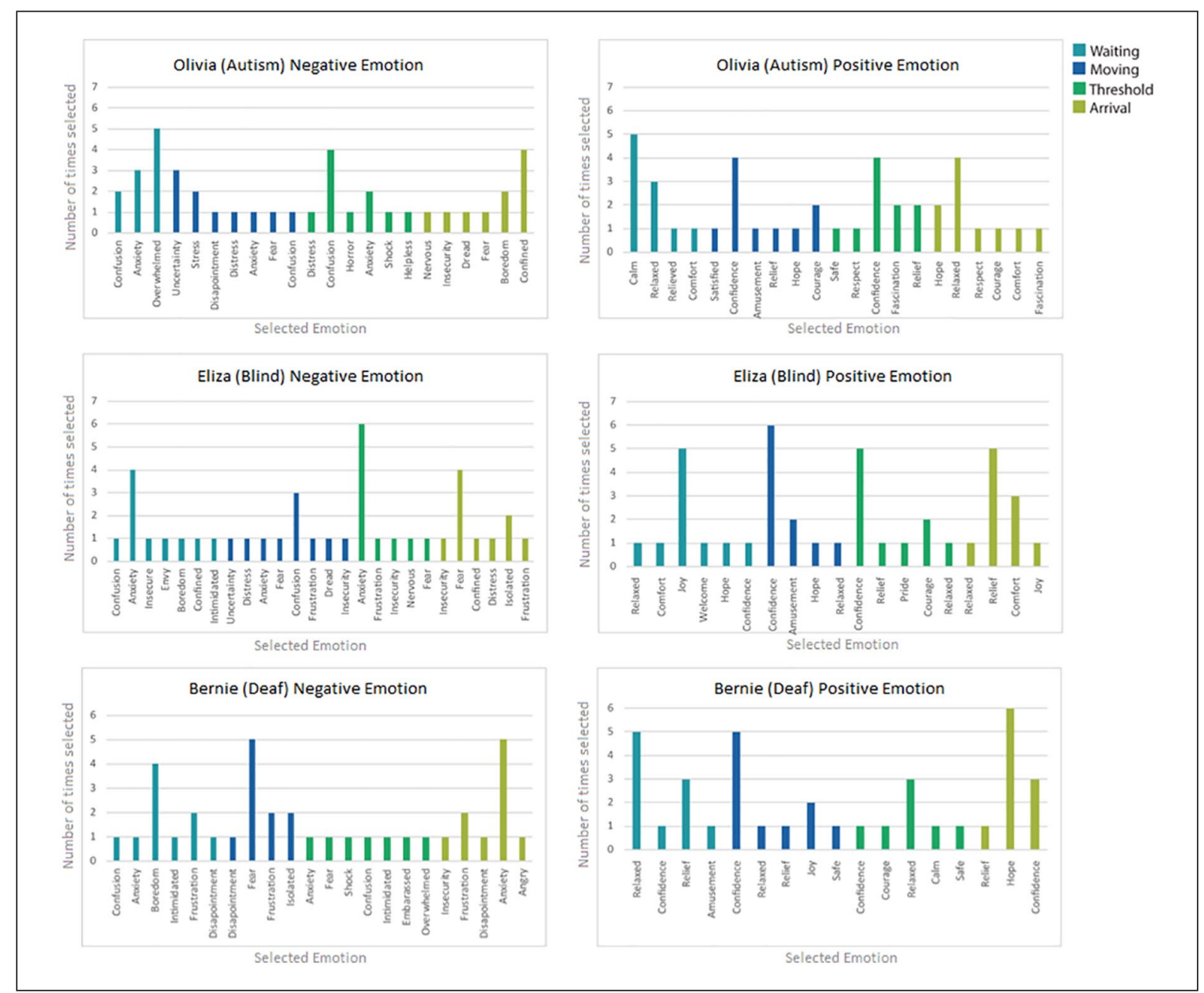

Figure 6. Emotions experienced by personas at each stage.

Following the experiment, design participants were surveyed regarding the importance of sensory impairment in influencing the emotions of the architecture. Ninety per cent of them identified a high relationship between design variations and emotional response.

\section{Discussion}

This research adopted an exploratory approach using a strategy of participatory processes to communicate design input from the primary occupants (impaired patients) to the designers, during potentially all phases of the design. It examined how VR could be used to understand the phenomenology and qualities of these interstitial health care places in relation to impairments, in particular those typically experienced by ED users. The experiments undertaken in this research were successful in achieving this aim despite limitations of available technology and stereotypical public opinion. VR is a relatively new technology, which was met with mixed opinion when exploring disability. Concerns were raised that it might be too insensitive to simulate disability. 
Three subject disabilities were chosen in order to give a broad idea of how the technology might work with different conditions. Choosing three impairments rather than one meant that the final simulations were not as detailed and refined as they could have been. For example, a greater degree of specificity with respect to the impairments simulated may establish the impairment as a set of different impairments (such as macular degeneration or glaucoma for those with visual impairments) and may require a simulation tool to be adequately flexible in representing these effectively. Similarly, the method for comparing the emotional responses of the non-impaired subjects to those with impairments was undertaken using an informal comparison of selected adjectives. In order to establish the effectiveness of the tool and validate the process, a more robust quantitative analysis is required. In addition, due to time constraints, it was not possible to look at less tangible constraints, such as cultural identity, language and understanding, or how these might be approached within the design. All these leave a clear path as to what further research on this topic might be able to achieve.

This research had several challenges that had to be overcome, largely revolving around a lack of understanding of the need of this type of research and the desire for the impaired users, who both wanted to make it happen and wanted to be a part of the research. All too often, disabled participants are portrayed as weak, vulnerable or sick individuals rather than active, concerned and interested members of society. The research gave a voice to people who have been previously ignored in architecture or only considered in the end stages of design, often with specific attention to accessibility only.

The research employed new technologies and explored their use in ways that had not previously been reported on. It provided positive results, suggesting that we can design for those with impairment and their individual emotional experiences, and that VR can be a successful way to do this. It has provided a sound starting point for future research to build upon.

The use of VR, however, is not without limitations. ${ }^{36}$ For example, our research found that the visually impaired people's preference was for sound reduction, indicating a preference for carpeting which contradicts other research seeking more sound reflection over silence since those with visual impairments derive information from sound created by reflection of solid and void. This needs further testing on a wider group of participants to validate the accuracy and clarity of the depicted impairments and their emotional response. ${ }^{37}$ The re-designed space that will evolve from the experiment will also require similar validation.

VR is limited in its simulation of sensory abilities as the programme is currently unable to simulate the experiences of smell and taste. Similarly, the haptic experience is limited to a visual element and cannot simulate the experience of changes to airflows, surface temperatures, or the tactile feel of a surface. Within the realm of the VR experience, further limitations result from the access to the software used and what the programme 'Fuzor' was able to accomplish. Within the simulations, the ability to move fluidly when transitioning from one space to another was limited as was the experience of the moment of moving through a threshold. The simulation also strayed from a true ED experience by not being surrounded by the movement of other people. The experience of other people in this research was reduced to stationary figures and an audio component. Similarly, the simulations were also limited in the way they were able to mimic the impairments of the personas, only being able to simulate visual and auditory aspects of their impairments.

\section{Conclusion}

Impairment is a complex phenomenon, reflecting the interaction between the features of a person's body and the features of the society in which he or she lives. People facing impairment are not simply passive users of services and buildings but can offer something powerful to architects and other building environment professionals if they are included and considered from the beginning phases of the design process. EDs have one of the most diverse groups of users, experiencing permanent or temporary impairments that in the past have not been considered with respect to the design. 
This article examined the use of VR as a technology to reveal and simulate the experiences of three different personas in this narrative. It also examined how design variables relating to the perceptual systems can influence the phenomenology of these spaces and therefore human emotion. The research further found that despite limitations, there is significant potential for the use of VR and participatory processes, working with both people facing impairment and people in the general public in order to revise the design of these spaces. By implementing such design methods, architects may be able to reduce distress, anxiety, delirium and high blood pressure of patients.

Participatory processes are a well-established approach to design, which considers the users at the core of the design process. In this study, participatory processes were employed in three stages. First is in the development of personas where people with impairments assisted in the development of the VR experience to enrich and elaborate on their lived experience of being in the space. Second, a participatory process was used with health care providers to better understand the ED experience and processes involved. Third, the final participants using VR as a design tool engaged directly with the impairment, allowing for empathetic design through an indirect end-user participation in the design. The participatory approach allowed VR simulations to be developed, representing different patients and their unique sensory conditions in order to examine how these conditions alter the critical phenomenological and emotional experiences within the 'between-moments' of the ED. The results confirm that different design variables have a significant influence on participant's emotions and that by adjusting these qualities with VR, the architect can explore and ultimately influence the way different users will experience the space, allowing an empathetic approach to design. Conventional participatory methods such as focus group interviews or questionnaires are too general for the specific stakeholders of emergency and health care spaces, and a more focussed and fine-grained approach is required.

VR can become an important medium in which a variety of participants can understand and experience the sensory conditions of others. It can allow others to inhabit the space in each stage of design, providing a heightened interface that can improve usability, simplicity and intelligibility of the architecture. It acknowledges any design process that involves the participation of patients' needs to elicit their needs by considering characteristics such as their sensory conditions and their background, as well as the simple pragmatics of moving through a space. 'Communication [thereby] becomes a continuous process of perspective, conceptualisation and information exchange, always requiring interpretation and translation of both the designers and users' ${ }^{38}$ The VR tool improves the knowledge of the emotional response made by certain design parameters, and consequently allows health care professionals and patients a chance to affect the design developments and outcomes without the lengthy process often associated with traditional participatory design methods.

Finally, the results demonstrated that the use of VR as a means of revealing and understanding human existence within space is valuable for interpreting the phenomenology of narrative and space, and how different design variables can evoke emotive responses in order to support human activities through interaction. The research confirms that VR can bridge the gap between philosophy and technology in order to design 'smarter' health care 'between-moments'.

\section{Declaration of conflicting interests}

The author(s) declared no potential conflicts of interest with respect to the research, authorship and/or publication of this article.

\section{Funding}

The author(s) received no financial support for the research, authorship and/or publication of this article.

\section{ORCID iD}

Bruno Marques (iD https://orcid.org/0000-0002-4761-8225 


\section{References}

1. Tenbensel T, Chalmers L, Jones P, et al. New Zealand's emergency department target-did it reduce ED length of stay, and if so, how and when? BMC Health Serv Res 2017; 17(1): 678.

2. New Zealand Herald. Emergency departments overwhelmed by patients, not meeting targets, www.nzherald.co.nz/ nz/news/article.cfm?c id=1\&objectid=12096563 (2018, accessed 15 October 2018).

3. Forero R, McCarthy S and Hillman K. Access block and emergency department overcrowding. Crit Care 2011; 15(2): 216.

4. Ministry of Health. Emergency department use 2014/15. Wellington: New Zealand Government, 2016.

5. Statistics New Zealand. Wellbeing statistics 2018. Wellington: Statistics New Zealand, 2018.

6. Forero R, Hillman KM, McCarthy S, et al. Access block and ED overcrowding. Emerg Med Australas 2010; 22(2): $119-135$.

7. Braitberg G. Emergency department overcrowding: dying to get in? Med J Aust 2007; 187(11/12): 624-625.

8. Schull MJ, Szalai JP, Schwartz B, et al. Emergency department overcrowding following systematic hospital restructuring trends at twenty hospitals over ten years. Acad Emerg Med 2001; 8(11): 1037-1043.

9. Mohsin M, Forero R, Ieraci S, et al. A population follow-up study of patients who left an emergency department without being seen by a medical officer. Emerg Med J 2007; 24(3): 175-179.

10. Forster AJ, Murff HJ, Peterson JF, et al. The incidence and severity of adverse events affecting patients after discharge from the hospital. Ann Intern Med 2003; 138(3): 161-167.

11. Sprivulis PC, Da Silva JA, Jacobs IG, et al. The association between hospital overcrowding and mortality among patients admitted via Western Australian emergency departments. Med J Aust 2006; 184(5): 208-212.

12. Ulrich RS. Essay: evidence-based health-care architecture. Lancet 2006; 368: S38-S39.

13. Heylighen A, Van Doren C and Vermeersch PW. Enriching our understanding of architecture through disability experience. Open House Int 2013; 38(1): 7-19.

14. Ou L, Young L, Chen J, et al. Discharge delay in acute care: reasons and determinants of delay in general ward patients. Aust Health Rev 2009; 33(3): 513-521.

15. Blumberg R and Devlin AS. Design issues in hospitals: the adolescent client. Environ Behav 2006; 38(3): $293-317$.

16. Drettakis G, Roussou M, Martinez A, et al. Design and evaluation of a real-world virtual environment for architecture and urban planning. Presence-Teleop Virt 2007; 16(3): 318-332.

17. Mobach MP. Do virtual worlds create better real worlds? Virtual Real 2008; 12(3): 163-179.

18. Frost $\mathrm{P}$ and Warren P. Virtual reality used in a collaborative architectural design process. In: IEEE conference on information visualization. An international conference on computer visualization and graphics (ed E Banissi, $\mathrm{M}$ Bannatyne, C Chen, et al.), London, 19-21 July 2000, pp. 568-573. Los Alamitos, CA: IEEE Computer Society.

19. Chicchi Giglioli IA, Pravettoni G, Sutil Martín DL, et al. A novel integrating virtual reality approach for the assessment of the attachment behavioral system. Front Psychol 2017; 8: 959.

20. Marín-Morales J, Higuera-Trujillo JL, Greco A, et al. Affective computing in virtual reality: emotion recognition from brain and heartbeat dynamics using wearable sensors. Sci Rep 2018; 8: 13657.

21. Alcañiz M, Baños R, Botella C, et al. The EMMA Project: emotions as a determinant of presence. Psychnol J 2003; 1(2): 141-150.

22. Baños RM, Liaño $\mathrm{V}$, Botella $\mathrm{C}$, et al. Changing induced moods via virtual reality. In: International conference on persuasive technology, Eindhoven, 18-19 May 2006, pp. 7-15. Berlin: Springer.

23. Baños RM, Etchemendy E, Castilla D, et al. Positive mood induction procedures for virtual environments designed for elderly people. Interact Comput 2012; 24(3): 131-138.

24. Zhao Y, Bennett CL, Benko H, et al. Enabling people with visual impairments to navigate virtual reality with a haptic and auditory cane simulation. In: Proceedings of the 2018 CHI conference on human factors in computing systems, Montreal, QC, Canada, 21-26 April 2018, paper no. 116, pp. 1-14. New York: Association for Computing Machinery.

25. Lécuyer A, Mobuchon P, Mégard C, et al. HOMERE: a multimodal system for visually impaired people to explore virtual environments. In: Proceedings of the IEEE virtual reality 2003, Washington, DC, 22-26 March 2003, pp. 251-258. Los Alamitos, CA: IEEE Computer Society.

26. Naz A, Kopper R, McMahan RP, et al. Emotional qualities of VR space. In: 2017 IEEE virtual reality (VR), Los Angeles, CA, 18-22 March 2017, pp. 3-11. Los Alamitos, CA: IEEE Computer Society. 
27. Naz A. Interactive living space design for neo-nomads: anticipation through spatial articulation. In: Nadin M (ed.) Anticipation across disciplines. Cham: Springer, 2016, pp. 393-403.

28. Carr BG, Kaye AJ, Wiebe DJ, et al. Emergency department length of stay: a major risk factor for pneumonia in intubated blunt trauma patients. $J$ Trauma 2007; 63(1): 9-12.

29. Franz G, Von Der Heyde M and Bülthoff HH. An empirical approach to the experience of architectural space in virtual reality: exploring relations between features and affective appraisals of rectangular indoor spaces. Automat Constr 2005; 14(2): 165-172.

30. Hershberger RG and Cass RC. Predicting user responses to buildings. In: Nassar J (ed.) Environmental aesthetics: theory, research and applications. Cambridge: Cambridge University Press, 1988, pp. 195-211.

31. Henry D and Furness T. Spatial perception in virtual environments: evaluating an architectural application. In: Proceedings of IEEE virtual reality annual international symposium, Seattle, WA, 18-22 September 1993, pp. 33-40. Los Alamitos, CA: IEEE Computer Society.

32. Ates HC, Fiannaca A and Folmer E. Immersive simulation of visual impairments using a wearable see-through display. In: Proceedings of the ninth international conference on tangible, embedded, and embodied interaction, Stanford, CA, 16-19 January 2015, pp. 225-228. New York: Association for Computing Machinery.

33. Desmet P. Designing emotions. Delft: Department of Industrial Design, Delft University of Technology, 2002.

34. Küller R, Ballal S, Laike T, et al. The impact of light and colour on psychological mood: a cross-cultural study of indoor work environments. Ergonomics 2006; 49(14): 1496-1507.

35. Harkness R, McIntosh J and Marques B. Using virtual reality and participatory processes to design interstitial healthcare places. In: International conference of the architectural (eds. Rajagopalan P and Andamon M), Melbourne, Australia, 28 November-1 December 2018, pp. 657-664. Melbourne, VIC, Australia: RMIT University.

36. McIntosh J, Rodgers M, Marques B, et al. The use of VR for creating therapeutic environments for the health and wellbeing of military personnel, their families and their communities. J Dig Landsc Archit 2019; 1(4): 185-195.

37. Hull RB IV and Harvey A. Explaining the emotion people experience in suburban parks. Environ Behav 1989; 21(3): 323-345.

38. Bruno F and Muzzupappa M. Product interface design: a participatory approach based on virtual reality. Int J HumComput St 2010; 68(5): 254-269. 